\title{
DIFFERENTIABLE INTRINSIC FUNCTIONS OF COMPLEX MATRICES ${ }^{1}$
}

\author{
R. F. RINEHART
}

Introduction. Let $\mathfrak{A}$ be a finite dimensional linear associative algebra with identity over the real or complex field $\mathcal{F}$ with basis $e_{1}, \cdots, e_{r}$. A function $F(x)$ with domain and range in $\mathfrak{A}$ may be written as $F(x)=\sum_{i=1}^{r} \eta_{i} e_{i}$, where $x=\sum_{i=1}^{r} \xi_{i} e_{i}$ and $\eta_{i}=\eta_{i}\left(\xi_{1}, \cdots, \xi_{r}\right), \xi_{i}, \eta_{i} \in \mathcal{F}$. $F(x)$ is called Hausdorff-differentiable (HD) at an element $a=\sum \alpha_{i} e_{i}$ $\in \mathfrak{A}$ if (1) the functions $\eta_{i}\left(\xi_{1}, \cdots, \xi_{r}\right)$ have first order partial derivatives with respect to each $\xi_{j}$ at $\left(\alpha_{1}, \cdots, \alpha_{r}\right)$ and (2) if the differential of $F(x)$,

$$
\left.d F(x)]_{x=a}=\sum_{i, j=1}^{r} \frac{\partial \eta_{i}}{\partial \xi_{j}} e_{i} d \xi_{j}\right]_{x=a},
$$

is expressible in the form $d F(x)=\sum_{j=1}^{k} P_{j} d x Q_{j}$ where $d x=\sum d \xi_{i} e_{i}$ and the $P_{j}, Q_{j} \in \mathfrak{Y}$ and are independent of $d x[2 ; 3 ; 8]$.

The Hausdorff derivative of an HD function [3] is defined to be the "differential coefficient" $\sum_{j=1}^{\boldsymbol{k}} P_{j} Q_{j}$.

It is well known $[8 ; 9]$ that condition (2) is automatically fulfilled if $\mathfrak{A}$ is normal simple, in particular if $\mathfrak{A}$ is the algebra $\mathfrak{M}_{C}^{n}$ of $n \times n$ matrices over the complex field $\mathfrak{e}$.

A function $F$ with domain and range in $\mathfrak{A}$ is called intrinsic [6] if for every automorphism or antiautomorphism $\Omega$ of $A$ which leaves $\mathcal{F}$ elementwise invariant, $F(\Omega a)=\Omega F(a)$. In [7] continuous intrinsic functions $F$ on $\mathfrak{M}_{C}^{n}$ have been essentially characterized as the functions arising from continuous, complex valued, stem functions $f\left(\lambda, \sigma_{1}, \cdots, \sigma_{n-1}\right)$ of $n$ complex variables, in the following way. Let $f\left(\lambda, \sigma_{1}, \cdots, \sigma_{n-1}\right)$ be defined at the eigenpoints of $A \in \mathfrak{M}_{C}^{n}$, $\left(\lambda_{i}[A], \sigma_{1}[A], \cdots, \sigma_{n_{-1}}[A]\right), i=1, \cdots, n$, where $\lambda_{i}[A]$ is an eigenvalue of $A$ and $\sigma_{j}[A]$ is the $j$ th elementary symmetric function of the eigenvalues of $A$. Let $f_{A}(\lambda)$ denote the function $f\left(\lambda, \sigma_{1}[A], \cdots\right.$, $\left.\sigma_{n-1}[A]\right)$ of the single complex variable $\lambda$, and let $f_{A}(\lambda)$ be analytic in a $\lambda$-neighborhood of each repeated eigenvalue of $A$. Then $F(A)$ is given by $f_{A}(A)$, where $f_{A}(A)$ is formed from $f_{A}(\lambda)$ according to any of the classical equivalent ways of extending a function of a single complex variable to matrices [4]. Functions $F$ obtained from stem functions $f\left(\lambda, \sigma_{1}, \cdots, \sigma_{n-1}\right)$ in this way are called $n$-ary functions. 1960.

Presented to the Society, September 1, 1960; received by the editors June 29,

${ }^{1}$ Supported by the Office of Ordnance Research, U. S. Army. 
Thus, the most general intrinsic function on $\mathfrak{M}_{C}^{n}$ defined in a neighborhood of $A$ and continuous at $A$ (with an additional assumption in case of argument matrices with repeated eigenvalues) is given by an $n$-ary function whose scalar stem function is continuous at each eigenpoint of $A$.

Primary functions, on $\mathfrak{M}_{C}^{n}$, i.e. those arising from the classical extension to $\mathfrak{M}_{C}^{n}$ of functions of a single complex variable, are the special cases of $n$-ary functions wherein the stem function is independent of the parameters $\sigma_{1}, \cdots, \sigma_{n-1}$.

The purpose of the present paper is to establish a relationship between the property of Hausdorff differentiability of intrinsic functions on $\mathfrak{M}_{C}^{n}$ and differentiability properties of the stem function $f\left(\lambda, \sigma_{1}, \cdots, \sigma_{n-1}\right)$, and to obtain an explicit formula for the Hausdorff derivative of an intrinsic function on $\mathfrak{M}_{C}^{n}$ in terms of derivatives of the stem function.

2. Intrinsic $H D$ functions at matrices with distinct eigenvalues. $\mathfrak{M}_{C}^{n}$ is a normed ring, (e.g. norm $A=\max _{r, s}\left|a_{r s}\right|$ ) thus a metric topological space in the metric induced by the norm. The concepts of limit and neighborhood are therefore well defined and the usual associated processes of elementary analysis are valid. The principal result of this section may be stated as:

Theorem 2.1. Let $A$ be a matrix of $\mathfrak{M}_{C}^{n}$ with distinct eigenvalues and let $F$ be an intrinsic function defined and $H D$ in a neighborhood $\mathfrak{N}$ of $A$. Then there exists a neighborhood of $A, \mathfrak{N}^{\prime} \subset \mathfrak{N}$, such that $F$ is an n-ary function in $\mathfrak{N}^{\prime}$, and the associated stem function $f\left(\lambda, \sigma_{1}, \cdots, \sigma_{n-1}\right)$ possesses first order partial derivatives with respect to each argument at each eigenpoint $\left(\lambda[X], \sigma_{1}[X], \cdots, \sigma_{n-1}[X]\right)$, for $X \in \mathfrak{N}^{\prime}$.

Proof. As already noted, the assumption that $F$ is HD in $\mathfrak{N}$ is merely the assumption that the elements of $F(X), X=\left(\xi_{r s}\right)$, possess first partial derivatives with respect to the $\xi_{r s}$ at $\left(\xi_{r s}\right)_{0}=X_{0} \in \mathfrak{R}$, since $\mathfrak{M}_{C}^{n}$ is normal simple.

Let $\Phi$ denote the multiple mapping of $\mathfrak{M}_{C}^{n}$ onto the complex Euclidean space $\varepsilon_{C}^{n}$ wherein $X \in \mathfrak{M}_{C}^{n}$ is mapped into the eigenpoints $\left(\lambda_{i}[X], \sigma_{1}[X], \cdots, \sigma_{n-1}[X]\right), i=1, \cdots, n$. Since $A$ has distinct eigenvalues, and since $\Phi$ is a continuous and open mapping [7], $\exists$ a neighborhood of $A, \mathfrak{N}^{\prime} \subset \mathfrak{R}$ such that (1) $X \in \mathfrak{N}^{\prime} \Rightarrow X$ has distinct eigenvalues, (2) the eigenpoints $\left(\lambda_{i}[X], \sigma_{1}[X], \cdots, \sigma_{n-1}[X]\right)$ lie in $n$ disjoint neighborhoods $\Gamma_{i}$ of $\varepsilon_{C}^{n}$, each containing just one eigenpoint of $X$ for $X \in \mathfrak{R}^{\prime}$, and (3) the eigenvalues $\lambda_{i}[X]$ lie in $n$ disjoint neighborhoods $\delta_{i}$ of the complex plane, each containing just one eigenvalue of $X$ for $X \in \mathfrak{N}^{\prime}$. Since $F$ is defined and HD in $\mathfrak{R}^{\prime}$ it is continuous in 
$\mathfrak{N}^{\prime}$. Since $F$ is intrinsic, $F$ is an $n$-ary function in $\mathfrak{N}^{\prime}$, and the associated stem function $f\left(\lambda, \sigma_{1}, \cdots, \sigma_{n-1}\right)$ is defined and continuous in the corresponding open set $U \Gamma_{i}$ of $\varepsilon_{C}^{n}$, containing the eigenpoints $\left(\lambda_{i}[A], \sigma_{1}[A], \cdots, \sigma_{n-1}[A]\right), i=1, \cdots, n,[7]$.

Let $P$ be a matrix of $\mathfrak{M}_{C}^{n}$ such that $P^{-1} A P=D=\operatorname{diag}\left[\alpha_{11}, \cdots, \alpha_{n n}\right]$ is diagonal. The similarity transformation by $P$ on $\mathfrak{M}_{C}^{n}$ is a biunique, continuous open mapping of $\mathfrak{M}_{C}^{n}$ onto itself, and the neighborhood $\mathfrak{N}^{\prime}$ is transformed into $P^{-1} \mathfrak{N}^{\prime} P=\widetilde{\mathfrak{N}}$, a neighborhood of $D$. By the intrinsic condition, $F$ is defined in $\tilde{\mathfrak{R}}$, and by the invariance of the property of Hausdorff differentiability under change of basis [8], the function $F$ is HD in $\tilde{\mathfrak{R}}$. The open sets $\Gamma_{i}$ of $\varepsilon_{C}^{n}$ associated with $\mathfrak{\Re}^{\prime}$ remain invariant under the similarity transformation by virtue of the invariance of the characteristic equations of the matrices of $\mathfrak{M}_{C}^{n}$ under similarity transformation.

Let $\mathfrak{D}$ be the set of diagonal matrices of $\tilde{\mathfrak{A}}$. $\mathfrak{D}$ is an open set in the set of all diagonal matrices and $\mathfrak{D}$ contains $D$. Since $F$ is an $n$-ary function in $\widetilde{\mathfrak{R}}$ it maps diagonal matrices into diagonal matrices [7]. Hence the restriction of $F$ to $\mathfrak{D}$ is of the form

$$
\begin{aligned}
F\left(\operatorname { d i a g } \left[\xi_{11}, \xi_{22},\right.\right. & \left.\left.\cdots, \xi_{n n}\right]\right) \\
& =\operatorname{diag}\left[\eta_{11}\left(\xi_{11}, \cdots, \xi_{n n}\right), \cdots, \eta_{n n}\left(\xi_{11}, \cdots, \xi_{n n}\right)\right] \\
& =\operatorname{diag}\left[f\left(\xi_{11}, \sigma_{1}, \cdots, \sigma_{n-1}\right), \cdots, f\left(\xi_{n n}, \sigma_{1}, \cdots, \sigma_{n-1}\right)\right],
\end{aligned}
$$

where $f\left(\lambda, \sigma_{1}, \cdots, \sigma_{n-1}\right)$ is the stem function of the $n$-ary function $F$, and the parameters $\sigma_{i}$ are the elementary symmetric functions of the $\xi_{i i}$. The $\eta_{i i}$ are the diagonal component functions of $F(X)$ evaluated at $\xi_{r s}=0, r \neq s$, and hence are differentiable functions of the $\xi_{i i}$ on the open set $\delta_{1} \times \delta_{2} \times \cdots \times \delta_{n}=\theta$, the cartesian product of the $\delta_{i}$, for $\operatorname{diag}\left[\xi_{11}, \cdots, \xi_{n n}\right] \in \mathfrak{D}$. The $\eta_{i i}$ are therefore analytic functions of the $n$ complex variables $\xi_{i i}$ in $\theta$ [1]. To show that $f\left(\lambda, \sigma_{1}, \cdots, \sigma_{n-1}\right)$ possesses first partial derivatives with respect to its arguments in $U \Gamma_{i}$ it is sufficient [1] to show that the Jacobians

$$
J_{j}=J_{j}\left(\lambda, \sigma_{1}, \cdots, \sigma_{n-1} ; \xi_{11}, \cdots, \xi_{n n}\right)
$$

of the transformations $\tau_{j}$ :

$$
\begin{aligned}
\lambda & =\xi_{j j} \\
\sigma_{1} & =\sum_{i=1}^{n} \xi_{i i} \\
\vdots & \vdots \\
\sigma_{n-1} & =\sum_{i=1}^{n} \frac{\xi_{11} \xi_{22} \cdots \xi_{n n}}{\xi_{i i}}
\end{aligned} \quad j=1,2, \cdots, n
$$


are each different from zero for $\left(\lambda, \sigma_{1}, \cdots, \sigma_{n-1}\right) \in \Gamma_{j}$. Each of the $\lambda, \sigma_{1}, \cdots, \sigma_{n-1}$ is obviously an analytic function of the $\xi_{i i}$ in $\theta$, and the partial derivatives occurring in $J_{j}$ are easily computed to be:

$$
\begin{aligned}
\frac{\partial \lambda}{\partial \xi_{i i}} & =\delta_{i j} \\
\frac{\partial \sigma_{1}}{\partial \xi_{i i}} & =1 \\
\frac{\partial \sigma_{2}}{\partial \xi} & =\sigma_{1}-\xi_{i i} \\
& \vdots \\
\frac{\partial \sigma_{m}}{\partial \xi_{i i}} & =\sigma_{m-1}-\xi_{i i} \sigma_{m-2}+\cdots+(-1)^{m-2} \xi_{i i}^{m-2} \sigma_{1}+(-1)^{m-1} \xi_{i i}^{m-1}, m<n,
\end{aligned}
$$

whence,

$$
J_{j}=\left|\begin{array}{cccccc}
0 & 0 & \cdots & 1 & \cdots & 0 \\
1 & 1 & \cdots & 1 & \cdots & 1 \\
\sigma_{1}-\xi_{11} & \sigma_{1}-\xi_{22} & \cdots & \sigma_{1}-\xi_{j j} & \cdots & \sigma_{1}-\xi_{n n} \\
\vdots & \vdots & & \vdots & & \vdots \\
\frac{\partial \sigma_{n-1}}{\partial \xi_{11}} & \frac{\partial \sigma_{n-1}}{\partial \xi_{22}} & \cdots & \frac{\partial \dot{\sigma}_{n-1}}{\partial \xi_{j j}} & \cdots & \frac{\partial \sigma_{n-1}}{\partial \xi_{n n}}
\end{array}\right| .
$$

Multiplying row 2 by $-\sigma_{i}$ and adding to row $i+2, i=1, \cdots, n-2$, then multiplying row 3 by $-\sigma_{i}$ and adding to row $i+3, i=1, \cdots$, $n-3$, then multiplying row 4 by $-\sigma_{i}$ and adding to row $i+4, i=1$, $\cdots, n-4$, etc., yields

$$
J_{j}=(-1)^{n-2}\left|\begin{array}{cccccc}
0 & 0 & \cdots & 1 & \cdots & 0 \\
1 & 1 & \cdots & 1 & \cdots & 1 \\
\xi_{11} & \xi_{22} & \cdots & \xi_{j j} & \cdots & \xi_{n n} \\
\xi_{11} & \xi_{22}^{2} & \cdots & \xi_{j j}^{2} & \cdots & \xi_{n n}^{2} \\
\vdots & \vdots & & \vdots & & \vdots \\
\vdots & \vdots & & \vdots & \\
\xi_{11}^{n-2} & \xi_{22}^{n-2} & \cdots & \xi_{j j}^{n-2} & \cdots & \xi_{n n}^{n-2}
\end{array}\right|
$$

which is a Vandermonde determinant of order $n-1$ and therefore different from zero for all $j$ since the $\xi_{i i}$ are distinct for all 
$\left(\xi_{11}, \cdots, \xi_{n n}\right) \in \theta$. This proves the theorem.

REMARK. Since mere continuity of $F$ nearly insures the existence of the partial derivative of $f\left(\lambda, \sigma_{1}, \cdots, \sigma_{n-1}\right)$ with respect to $\lambda$ at repeated eigenpoints [7], there is some reason to feel that the conclusion of Theorem 2.1 might be valid without the assumption of distinct eigenvalues essential to this proof. A settling of this question would be of interest.

3. $n$-ary functions with differentiable stem functions. The problem converse to that of Theorem 2.1, will now be investigated. Here the restriction of distinct eigenvalues will not be necessary.

Theorem 3.1. Let $f\left(\lambda, \sigma_{1}, \cdots, \sigma_{n-1}\right)$ be a function of $n$ complex variables defined on an open set $\Gamma$ of $\varepsilon_{C}^{n}$ and possessing first partial derivatives with respect to each argument at each point of $\Gamma$. Then the $n$-ary function $F(X)=f\left(X, \sigma_{1}[X], \cdots, \sigma_{n-1}[X]\right)$ is defined on a corresponding open set $\Re$ of $\mathfrak{M}_{C}^{n}$ consisting of all matrices of $\mathfrak{M}_{C}^{n}$ whose eigenpoints lie in $\Gamma$, and $F(X)$ is intrinsic and $H D$ in $\Omega$.

Theorem 3.1 is a generalization of a theorem of Portmann [3] on primary functions, and the present proof will follow Portmann's approach with appropriate modifications.

Since at every point of $\Gamma f\left(\lambda, \sigma_{1}, \cdots, \sigma_{n-1}\right)$ has a partial derivative with respect to $\lambda, F(X)$ is defined at every matrix $X$ of $\Omega$ whether $X$ has distinct eigenvalues or not [7]. That $\Re$ is open and

$$
f\left(X, \sigma_{1}[X], \cdots, \sigma_{n-1}[X]\right)
$$

is intrinsic on $\Omega$ is proved in [7].

To show that $F(X)$ is HD at $A \in \Omega$ it is sufficient to show that the elements of $F(X)$ possess first partial derivatives with respect to the elements $\xi_{i j}$ of $X$ in a neighborhood of $A$. To this end we shall employ the Cauchy integral representation of $F(X)$.

By the continuity of the eigenvalues of a matrix as functions of the elements of a matrix and the continuity of the mapping $\Phi$ of $\S 2$, a neighborhood $\mathfrak{R}=\{X: \operatorname{norm}(X-A)<\epsilon, \epsilon>0$, real $\}$ of $A$ in $\mathfrak{M}_{C}^{n}$ can be so chosen that $\mathfrak{N} \subset \Re$ and the eigenvalues of matrices of $\mathfrak{R}$ lie in $k$ disjoint complex plane neighborhoods $\delta_{i}$ of the $k$ distinct eigenvalues of $A, 0<k \leqq n$. The eigenpoints of matrices of $\mathfrak{N}$ constitute an open subset of $\Gamma$. Let $\Delta_{i}$ be a set of simple closed curves in the complex plane which enclose the distinct eigenvalues of $A$ and each of which together with its interior is contained in the corresponding $\delta_{i}$. Let $\mathfrak{N}^{\prime}$ be a subneighborhood of $A, \mathfrak{N}^{\prime} \subset \mathfrak{N}$, such that the eigenvalues of matrices of $\mathfrak{R}^{\prime}$ are inside the $\Delta_{i}$. Then from [7], for any $X \in \mathfrak{N}^{\prime}$, 


$$
\begin{aligned}
F(X) & =f\left(X, \sigma_{1}[X], \cdots, \sigma_{n-1}[X]\right) \\
& =\frac{1}{2 \pi i} \int_{U_{\Delta_{i}}} f\left(\lambda, \sigma_{1}[X], \cdots, \sigma_{n-1}[X]\right)(\lambda I-X)^{-1} d \lambda .
\end{aligned}
$$

The element $F_{r s}$ of $F(X)$ is given by

$$
F_{r s}=\frac{1}{2 \pi i} \int_{U \Delta_{i}} f\left(\lambda, \sigma_{1}[X], \cdots, \sigma_{n-1}[X]\right) R_{r s}\left(\lambda, \xi_{i j}\right) d \lambda,
$$

where $R_{r s}\left(\lambda, \xi_{i j}\right)$ is the quotient of two polynomials in $\lambda$ and the $\xi_{i j}$, $i, j=1, \cdots, n$. Since for all $X$ in $\mathfrak{R}^{\prime}, \lambda I-X$ is nonsingular for any $\lambda$ on any $\Delta_{i}, R_{r s}$ is an analytic function of the $n^{2}$ complex variables $\xi_{i j}$, for $\left(\xi_{r s}\right) \in \mathfrak{N}^{\prime}$ and for every $\lambda$ on $U \Delta_{i}$, and is continuous in all variables for $X \in \mathfrak{N}^{\prime}$ and $\lambda$ on $U \Delta_{i}$. Further since $f\left(\lambda, \sigma_{1}, \cdots, \sigma_{n-1}\right)$ is an analytic function of $\lambda, \sigma_{1}, \cdots, \sigma_{n-1}$ in $\Gamma[1]$, and since $\sigma_{1}[X]$, $\cdots, \sigma_{n-1}[X]$ are each polynomials in, hence analytic functions of, the $\xi_{i j}$, then $f\left(\lambda, \sigma_{1}[X], \cdots, \sigma_{n-1}[X]\right)$ is an analytic function of the $\xi_{i j}$ for $X \in \mathfrak{N}^{\prime}$. Therefore the integrand of (3.2) is analytic in the $\xi_{i j}$, and continuous in the $\xi_{i j}$ and $\lambda$, for $X \in \mathfrak{R}^{\prime}, \lambda$ on $U \Delta_{i}$.

Hence by a well-known theorem for functions of two complex variables, equally valid for any number of complex variables [10], it follows that the $F_{r s}$ are analytic functions of the $\xi_{i j}$ for $X \in \mathfrak{R}^{\prime}$. Therefore $f\left(X, \sigma_{1}[X], \cdots, \sigma_{n-1}[X]\right)$ is HD in $\mathfrak{N}^{\prime} \supset A$, and therefore HD in $\Omega$, since $A$ was arbitrary in $\Omega$.

4. The Hausdorff derivative of an $n$-ary function. Portmann [3] has shown that the Hausdorff derivative of an HD function $F(X)$ on $\mathfrak{M}_{C}^{n}, X=\left(\xi_{r s}\right)$ is given by the sum of the partial derivatives of $F(X)$ with respect to the diagonal variables of $X$, i.e.

$$
F^{I}(X)=\sum_{i=1}^{n} \frac{\partial F(X)}{\partial \xi_{i i}} .
$$

This result enables an explicit formulation of the Hausdorff derivative of an HD $n$-ary function in terms of partial derivatives of its stem function.

TheOREM 4.1. Let $f\left(\lambda, \sigma_{1}, \cdots, \sigma_{n-1}\right)$ be defined, and possess a first partial derivative with respect to each argument, in an open set $\Gamma$ of $\mathcal{E}_{C}^{n}$. Let $\Re$ be the set of matrices of $\mathfrak{M}_{C}^{n}$ whose eigenpoints lie in $\Gamma$. Then the Hausdorff derivative of the $n$-ary function

$$
F(X)=f\left(X, \sigma_{1}[X], \cdots, \sigma_{n-1}[X]\right)
$$

exists everywhere in $\Omega$ and is again an intrinsic function in $\Re$ whose stem function is 


$$
\begin{aligned}
& f^{I}\left(\lambda, \sigma_{1}, \cdots, \sigma_{n-1}\right) \\
& \quad=f_{\lambda}\left(\lambda, \sigma_{1}, \cdots, \sigma_{n-1}\right)+\sum_{j=1}^{n-1}(n-j+1) \sigma_{j-1} f_{\sigma_{j}}\left(\lambda, \sigma_{1}, \cdots, \sigma_{n-1}\right),
\end{aligned}
$$

the subscripts denoting partial derivatives, and $\sigma_{0}$ being defined to be 1 .

Proof. Let $A$ be any matrix in $\Omega$ and let $\mathfrak{N}^{\prime} \subset \Omega$ and $\Delta_{i}$ be chosen as in the proof of Theorem 3.1. Then $F(X)$ for $X \in \mathfrak{N}^{\prime}$ is given by the Cauchy integral formula (3.1), and conditions permitting differentiation under the integral sign with respect to the parameters $\xi_{i j}$ are met [10]. Hence, employing well-known principles for differentiation of matric functions of scalar variables, and utilizing the differentiability of the stem function and of the $\sigma_{i}[X]$, Portmann's result yields, for all $X$ in $\mathfrak{N}^{\prime}$,

$$
\begin{aligned}
F^{I}(X)= & \sum_{j=1}^{n} \frac{\partial F(X)}{\partial \xi_{j j}} \\
= & \frac{1}{2 \pi i} \int_{\cup_{\Delta_{i}}} f\left(\lambda, \sigma_{1}[X], \cdots, \sigma_{n-1}[X]\right)(\lambda I-X)^{-2} d \lambda \\
& +\sum_{j=1}^{n-1} \frac{1}{2 \pi i} \int_{\cup_{\Delta_{i}}} f_{\sigma_{j}}\left(\lambda, \sigma_{1}[X], \cdots, \sigma_{n-1}[X]\right)(\lambda I-X)^{-1} d \lambda \\
& \cdot \sum_{k=1}^{n} \frac{\partial \sigma_{j}[X]}{\partial \xi_{k k}} .
\end{aligned}
$$

Since $f\left(\lambda, \sigma_{1}[X], \cdots, \sigma_{n-1}[X]\right)$ is analytic in $\lambda$ for $X \in \mathfrak{N}^{\prime}, \lambda=\lambda_{i}[X]$, $i=1, \cdots, n$, the first term of the right member of 4.1 is simply the $n$-ary function of $X$ whose stem function is $f_{\lambda}\left(\lambda, \sigma_{1}, \cdots, \sigma_{n-1}\right)$. Simplification of the remaining $n-1$ integrals rests upon

LEMMA 4.1.

$$
\sum_{k=1}^{n} \frac{\partial \sigma_{j}[X]}{\partial \xi_{k k}}=(n-j+1) \sigma_{j-1}[X], \quad j=1, \cdots, n,\left(\sigma_{0}(X)=1\right) .
$$

For $j=1$, the formula is obvious. For $j>1$, since $\sigma_{j}[X]$ is the sum of all $j$-rowed principal minor determinants of $X$, by the rule for differentiation of determinants $\sum_{k=1}^{n} \partial \sigma_{j}[X] / \partial \xi_{k k}$ will be the sum of a number of $(j-1)$-rowed principal minors of $X$, in which sum the minor with rows $r_{1}, \cdots, r_{j-1}$ of $X$ will occur once for each selection of $\xi_{k k}$ with $k \neq r_{1}, r_{2}, \cdots, r_{j-1}$, i.e. $n-j+1$ times. This establishes the lemma.

Thus, the last $n-1$ integrals of (4.1) become 


$$
\begin{array}{r}
\frac{1}{2 \pi i}(n-j+1) \sigma_{j-1}[X] \int_{U_{\Delta_{i}}} f_{\sigma_{j}}\left(\lambda, \sigma_{1}[X], \cdots, \sigma_{n-1}[X]\right)(\lambda I-X)^{-1} d \lambda, \\
j=1, \cdots, n-1 .
\end{array}
$$

Each of these integrals is an $n$-ary function of $X$ with stem function $(n-j+1) \sigma_{j-1} f_{\sigma_{j}}\left(\lambda, \sigma_{1}, \cdots, \sigma_{n-1}\right), j=1, \cdots, n-1$, proving Theorem 4.1 .

Theorem 4.1 when specialized to primary functions, i.e. $n$-ary functions whose stem functions are independent of $\sigma_{1}, \cdots, \sigma_{n-1}$, yields the known result $[\mathbf{3} ; \mathbf{5}]$.

Corollary 4.1.1. If $f(\lambda)$ is an analytic function in an open set of the complex plane, then the primary function $f(X)$ is $H D$ in the corresponding open set $\Omega$ of $\mathfrak{M}_{c}^{n}$ and the Hausdorff derivative of $f(X)$ is the primary function with stem function $f^{\prime}(\lambda)$.

The analyticity of $f\left(\lambda, \sigma_{1}, \cdots, \sigma_{n-1}\right)$, the analyticity of the $\sigma_{k}[X]$ in the $\xi_{i j}, i, j=1, \cdots, n$, and the $n$-ary character of the Hausdorff derivative of an HD $n$-ary function, yield

Corollary 4.1.2. An n-ary function defined in a neighborhood $\mathfrak{N}$ of $A \in \mathfrak{M}_{C}^{n}$ whose stem function is analytic in the corresponding $\mathcal{E}_{C}^{n}$ neighborhood of the eigenpoints of $A$, has successive Hausdorff derivatives of all orders in $\mathfrak{R}$. Each derivative is again an $n$-ary function on $\mathfrak{R}$.

Hausdorff differentiability implies [3] differentiability in the generalized difference quotient sense of [5], and the Hausdorff derivative is equal to the limit of that generalized difference quotient. Similar conclusions can consequently be drawn concerning these differential quotients.

\section{REFERENCES}

1. S. Bochner and W. T. Martin, Several complex variables, Princeton University Press, 1948.

2. F. Hausdorff, Zur Theorie der Systeme complexer Zahlen, Leipziger Berichte vol. $52(1900)$ pp. 43-61.

3. W. O. Portmann, A derivative for Hausdorff-analytic functions, Proc. Amer. Math. Soc. vol. 10 (1959) pp. 101-105.

4. R. F. Rinehart, The equivalence of definitions of a matric function, Amer. Math. Monthly vol. 62 (1955) pp. 395-414.

5. - Extension of the derivative concept for functions of matrices, Proc. Amer. Math. Soc. vol. 8 (1957) pp. 329-335.

6. - Elements of a theory of intrinsic functions on algebras, Duke Math. J. vol. 27 (1960) pp. 1-19.

7. - Intrinsic functions on matrices, Duke Math. J. vol. 28 (1961) pp. 291300. 
8. F. Ringleb, Beiträge zur Funktionentheorie in hyperkomplexen Systemen. I, Rend. Circ. Mat. Palermo vol. 57 (1933) pp. 311-340.

9. K. Shoda, Ein Kriterium für normale einfache hyperkomplexen Systeme, Proc. Imp. Acad. Japan, Tokyo vol. 10 (1934) pp. 195-197.

10. E. C. Titchmarsh, The theory of functions, Oxford University Press, 1947.

Duke UNIVERSITY AND

Case Institute of Technology

\section{A GENERALIZATION OF HIRZEBRUCH POLYNOMIAL AND COBORDISM DECOMPOSITION}

\section{YASURÔ TOMONAGA}

Introduction. In this paper we shall generalize the Hirzebruch polynomial and utilize it for the determination of cobordism components of a compact orientable and differentiable $4 k$-manifold.

1. Let $X^{4 k}$ be a compact orientable and differentiable $4 k$-manifold. According to Thom's theorem [1] such a manifold is "cobordant" with a polynomial of the complex projective spaces except torsion, i.e.

$$
X^{4 k} \approx \sum_{i_{1}+\cdots+i_{t}=k} A_{i_{1}+\cdots+i_{t}}^{k} P_{2 i_{1}}(c) \cdots P_{2 i_{t}}(c) \text { mod torsion }
$$

where $P_{i}(c)$ denotes the complex projective space of the complex dimension $i$ and $A$ 's denotes some rational numbers. The Hirzebruch polynomial is defined as follows [2]

$$
\prod_{i} \frac{\left(\gamma_{i}\right)^{1 / 2}}{\operatorname{tgh}\left(\gamma_{i}\right)^{1 / 2}}=\sum_{i=0}^{\infty} L_{i}\left(p_{1}, \cdots, p_{i}\right), \quad \sum_{i=0}^{\infty} p_{i}=\prod_{i}\left(1+\gamma_{i}\right)
$$

where $p_{i}$ denotes the Pontryagin class of dimension $4 i$. It is well known that

$$
L_{i}\left(p_{1}, \cdots, p_{i}\right)\left[P_{2 i}(c)\right]=1,
$$

from which we have

$$
L_{k}\left(p_{1}, \cdots, p_{k}\right)\left[X^{4 k}\right]=\text { index of } X^{4 k}=\sum_{i_{1}+\cdots+i_{t}=k} A_{i_{1} \cdots i_{t}}^{k} .
$$

We generalize (1.2) as follows:

\footnotetext{
Received by the editors September 9, 1960.
} 